\title{
COMMUNICATION
}

\section{Carcinome adénosquameux de la cavité buccale : A propos d'un cas. Et revue de la littérature}

\author{
Benat $\mathrm{G}^{1}$, Alice Cros $\mathrm{A}^{2}$, Sarini $\mathrm{J}^{3}$, Galissier $\mathrm{T}^{4}$, Barres $\mathrm{B}^{5}$, Sarah Cousty $\mathrm{S}^{6}$ \\ 1 - interne DESCO
}

Le carcinome adénosquameux (CAS) de la partie supérieure des voies aéro-digestives est une entité extrêmement rare avec une centaine de cas diagnostiqués jusqu'à présent, de diagnostic difficile dont l'origine histologique est controversée (origine salivaire ou épithéliale).

Une femme de 66 ans, sans antécédents médico-chirurgicaux particuliers, consulte pour des douleurs chroniques sublinguales. L'interrogatoire rapporte un tabagisme actif à 40PA, sans exogénose chronique. Les douleurs sublinguales évoluent par périodes depuis environ 4 ans. L'examen clinique de la zone douloureuse révèle une large plage érythémateuse du plancher postérieur droit, remontant sur le versant lingual de la crête mandibulaire édentée en distal de 44. Sur la plage érythémateuse siège une ulcération dans le sillon pelvi-lingual, entourée de zones kératosiques d'aspect blanchâtre, inhomogènes, plus ou moins épaisses. L'examen est douloureux, la lésion ne saigne pas au contact et la palpation est souple sans induration. L'examen fonctionnel lingual est normal, la déglutition et la phonation ne sont pas non plus perturbées. L'examen exo buccal est sans particularité, sans adénopathie, ni tuméfaction. La patiente est porteuse d'une prothèse amovible résine adaptée et dont le bord lingual n'est pas en contact avec la muqueuse du plancher.

Devant cet aspect inhomogène il est décidé de pratiquer une biopsie incisionnelle. L'analyse anatomopathologique de la biopsie pose le diagnostic de carcinome adénosquameux.

La patiente est donc adressée à un service de chirurgie ORL carcinologique.

Elle bénéficie dans le cadre du bilan préopératoire d'une pan endoscopie des voies aéro-digestives supérieures, d'un scanner cervico-facial et thoracique et d'une IRM cervico-faciale pour caractériser précisément la lésion. II n'y a pas de signe d'envahissement ganglionnaire ou métastatique. La lésion est classée cT1NOM0. Une prise en charge chirurgicale est décidée avec pelvimandibulectomie non interruptrice, curage cervical complet droit et reconstruction par lambeau myo-muqueux de buccinateur à pédicule postérieur. L'analyse anatomopathologique de la pièce opératoire confirme la nature adénosquameuse de la lésion. La patiente bénéficie par la suite d'une radiothérapie adjuvante compte tenu de l'agressivité de ce type de tumeur et d'une surveillance rapprochée devant le fort taux de récidive précoce aussi bien locale que locorégionale. L'origine de cette tumeur est toujours débattue avec une orientation plus forte vers une origine épithéliale. Elle se présente sous la forme d'un double contingent présent simultanément mais en quantité très variable. Une composante épithéliale le plus souvent superficielle pouvant être in situ ou invasive, avec des zones de kératinisation. Une composante glandulaire invasive avec 
engainements péri-nerveux et embols vasculaires fréquents, pouvant ou non être sécrétante, plus en profondeur et pouvant passer inaperçue lors d'une biopsie trop superficielle.

Une revue de la littérature permet de noter une atteinte masculine $(3: 1)$ avec un âge médian de survenue de 60 ans et un taux de survie à 5 ans compris entre 13 et $61 \%$ selon les études. Le carcinome adénosquameux est une tumeur rare et de mauvais pronostic, méconnue et de diagnostic difficile, qui nécessite une prise en charge globale et complète avec des marges d'exérèse larges, pour espérer améliorer la survie sans récidive.

1. Keelawat S, Liu CZ, Roehm PC, Barnes L. Adenosquamous carcinoma of the upper aerodigestive tract : A clinicopathologic study of 12 cases and review of the literature. American Journal of Otolaryngology. mai 2002;23(3):160-8.

2. Alos L, Castillo M, Nadal A, Caballero M, Mallofre C, Palacin A, et al. Adenosquamous carcinoma of the head and neck : criteria for diagnosis in a study of 12 cases. Histopathology. 2004;44(6):570-9.

3. Schick U, Pusztaszeri M, Betz M, Ghadjar P, Demiroz C, Kaanders JHAM, et al. Adenosquamous carcinoma of the head and neck : report of 20 cases and review of the literature. Oral Surgery, Oral Medicine, Oral Pathology and Oral Radiology. sept 2013;116(3):313-20. 